\title{
FLEXIBLE LINK END-POINT CONTROL BASED ON EXACT DYNAMIC INVERSION: EXPERIMENTAL RESULTS
}

\author{
Aurelio Piazzi \\ Dipartimento di Ingegneria dell'Informazione \\ Faculty of Engineering \\ University of Parma \\ Parma, Italy \\ Email: aurelio@ce.unipr.it
}

\author{
Antonio Visioli* \\ Dipartimento di Elettronica per l'Automazione \\ Faculty of Engineering \\ University of Brescia \\ Brescia, Italy \\ visioli@ing.unibs.it
}

\begin{abstract}
In this paper we present a new method for the point-to-point motion control of the end-point of a single flexible link manipulator. The technique is based on an exact system inversion procedure, that allows to define a suitable motion law for the hub in order to reduce the residual vibration at the end of the tip motion. Thus, the end-point control is actually performed in open-loop, therefore avoiding the use of a sensor to measure the actual tip position. Experimental results demonstrate the effectiveness of the approach and that the overall control system is inherently robust to modelling errors.
\end{abstract}

\section{INTRODUCTION}

In the last twenty years, flexible manipulators have attracted much attention because of the many advantages they ensure with respect to the conventional rigid ones. Indeed, they need less material, smaller actuators, less energy and at the same time they are capable to assure faster motions and a higher payload to arm weight ratio. However, as a drawback, the control design task is much more difficult because, despite the fact that good results can be achieved with a linearized model (see for example (Geniele et al., 1997)), many flexible modes are required to accurately model the system. In addition, the system is nonminimumphase, because the positioning sensor at the manipulator tip is non colocated with the actuator at the hub. As a consequence, a straightforward application of a standard inverse control strategy

\footnotetext{
*Address all correspondence to this author
}

leads to an internal instability of the system and its use is therefore not possible in practical cases.

To achieve a stable inversion, many solutions have been recently proposed. For example, the concept of integral manifold has been exploited in (Hashtrudi Zaad, 1996), whilst in (Geniele et al., 1995; Geniele et al., 1997) the principle of transmission zero assignment is adopted in an output feedback control strategy. The zero dynamics can also been stabilized by means of the output redefinition approach (Moallem et al., 1997; Talebi et al., 1999; Yang et al., 1999) or by considering a series expansion of the inverse of the nonminimum-phase zeros (Gross and Tomizuka, 1994). Finally, iterative learning control (Panzieri and Ulivi, 1995; De Luca et al., 1998) and neural networks (Talebi et al., 1998) have been also successfully applied.

In this paper we present a methodology to analitically perform an exact stable inversion of a linearized model of the system. Roughly stating, the overall technique consists of defining a priori, as a "transition polynomial" (Piazzi and Visioli, 2001a), the desired motion law of the robot tip and then determining, by means of an exact stable inversion procedure, the corresponding motion law of the motor at the hub. This motion planning approach has been already exploited for the robust control of minimum-phase mechanical systems, namely, for the residual vibration reduction in point-to-point motions of servopositioning systems endowed with an elastic transmission (Piazzi and Visioli, 2000).

In this context, the control of the end-effector position is actually performed in open-loop, whilst a closed-loop controller is applied to the motor in order to track the calculated motion law.

Copyright (C) 2001 by ASME 
This choice has been done because, although with the proposed dynamic inversion methodology it is possible to directly compute the motor torque to be applied to the motor and to implement a control scheme with a feedback of the tip position, in this way there is no need to measure the tip position itself. Moreover, the modelling task is simplified, as it is related only to the flexible link and therefore problems such as the motor friction can be handled at a control level, by using specific methodologies (Geniele et al., 1997). Despite the fact that an open-loop approach is adopted, the methodology appears to be very robust to modelling errors, as it is shown by the presented experiments where, although only one flexible mode has been considered in the linear model of the link, the residual oscillation of the tip is indeed very small.

The paper is organized as follows. In the next section the transfer function of a single flexible link is determined and some notation is introduced. Then, the dynamic inversion based methodology is exposed. Subsequently, the experimental setup is described and results are presented. Conclusions are drawn in the last section.

\section{TRANSFER FUNCTION OF A SINGLE FLEXIBLE LINK}

The single flexible link we consider is assumed to be a uniform beam, of length $h$ and mass density $\gamma$. One end of the link is connected to a motor and the other one is free. It is also assumed that the height of the link is much greater than its width, that is, the link moves and vibrates in the horizontal plane and the effects of the shear deformation are negligible. Considering a frame $\mathrm{X}-\mathrm{Y}$, defined such as the $\mathrm{X}$-axis always passes through the center of gravity of the link, the position of any point along the link can be written as:

$$
y(t)=x \theta(t)+w(x, t)
$$

where $\theta(t)$ is the rotation of the hub and $w(x, t)$ is the elastic deflection (see Figure 1). Using the assumed-modes approach (Meirovitch, 1967; Menini et al., 1996), the elastic deflection can be expressed by:

$$
w(x, t)=\sum_{i=1}^{n} q_{i}(t) \phi_{i}(x)
$$

where $q_{i}(t)$ is the generalized coordinate of the $i$ th mode and $\phi_{i}(x)$ is the normalized, clamped-free eigenfunction of the $i$ th mode:

$\phi_{i}(x)=c_{i}\left[\sin k_{i} x-\sinh k_{i} x-\frac{\sin k_{i} h+\sinh k_{i} h}{\cos k_{i} h+\cosh k_{i} h}\left(\cos k_{i} h-\cosh k_{i} h\right)\right]$ where the $k_{i}$ are the solutions to:

$$
\cos \left(k_{i} h\right) \cosh \left(k_{i} h\right)=-1
$$

and the $c_{i}$ are normalization constants to be chosen so that

$$
\int_{0}^{h} \phi_{i}(x)^{2} d x=1
$$

Starting from the Euler-Lagrange equations and following the derivations reported in (Wang and Vidyasagar, 1989; Wang and Vidyasagar, 1991; Geniele et al., 1995; Geniele et al., 1997), we can write the equations of the non linear model of the system in which it is assumed that the hub position control is ideal, i.e. the actual hub position is equal to the desired one. The verification of this assumption is facilitated in practical cases by the presence of a speed reducer (as the inertia torque exerted on the hub by the flexible modes, i.e. the rigid body modes of the system and the flexible ones are decoupled) and by an appropriate selection of the controller's gain which makes the rigid body modes to decay much more rapidly than the flexible ones (Geniele et al., 1995; Geniele et al., 1997). Thus, considering the hub position $\theta$ as input of the system and the tip position $y$ as output, it results:

$$
\mathbf{M q}+\mathbf{C} \dot{\mathbf{q}}+K_{1} \mathbf{q}=\dot{\theta}^{2} K_{\mathbf{2}} \mathbf{q}-\mathbf{m} \ddot{\theta}
$$

where:

$$
\mathbf{M}=\left[\begin{array}{cccc}
\gamma+M_{p} \phi_{1}^{2}(h) & M_{p} \phi_{1}(h) \phi_{2}(h) & \cdots & M_{p} \phi_{1}(h) \phi_{n}(h) \\
M_{p} \phi_{2}(h) \phi_{1}(h) & \gamma+M_{p} \phi_{2}^{2}(h) & \cdots & M_{p} \phi_{2}(h) \phi_{n}(h) \\
\vdots & \vdots & \vdots & \vdots \\
M_{p} \phi_{n}(h) \phi_{1}(h) & M_{p} \phi_{n}(h) \phi_{2}(h) & \cdots & \gamma+M_{p} \phi_{n}^{2}(h)
\end{array}\right]
$$

$$
\mathbf{C}=\left[\begin{array}{cccc}
c_{1} & 0 & \cdots & 0 \\
0 & c_{2} & \cdots & 0 \\
\vdots & \vdots & \vdots & \vdots \\
0 & 0 & \cdots & c_{n}
\end{array}\right]
$$

$$
\mathbf{K}_{\mathbf{1}}=\gamma\left[\begin{array}{cccc}
\omega_{1}^{2} & 0 & \cdots & 0 \\
0 & \omega_{2}^{2} & \cdots & 0 \\
\vdots & \vdots & \vdots & \vdots \\
0 & 0 & \cdots & \omega_{n}^{2}
\end{array}\right]
$$




$$
\begin{aligned}
& \mathbf{K}_{\mathbf{2}}=\left[\begin{array}{cccc}
\gamma+M_{p} \phi_{1}^{2}(h) & M_{p} \phi_{1}(h) \phi_{2}(h) & \cdots & M_{p} \phi_{1}(h) \phi_{n}(h) \\
M_{p} \phi_{2}(h) \phi_{1}(h) & \gamma+M_{p} \phi_{2}^{2}(h) & \cdots & M_{p} \phi_{2}(h) \phi_{n}(h) \\
\vdots & \vdots & \vdots & \vdots \\
M_{p} \phi_{n}(h) \phi_{1}(h) & M_{p} \phi_{n}(h) \phi_{2}(h) & \cdots & \gamma+M_{p} \phi_{n}^{2}(h)
\end{array}\right] \\
& \mathbf{m}^{T}=\left[\gamma \int_{0}^{h} \phi_{1} x d x+M_{p} h \phi_{1}(h) \quad \gamma \int_{0}^{h} \phi_{2} x d x+M_{p} h \phi_{2}(h) \cdots\right. \\
& \left.\cdots \gamma \int_{0}^{h} \phi_{n} x d x+M_{p} h \phi_{n}(h)\right]
\end{aligned}
$$

In the previous expressions, $M_{p}$ is the mass of the load, $\omega_{i}$ is the resonance frequency of the $i$ th clamped-free eigenfunction and the terms $c_{i} \dot{q}_{i}(i=1, \ldots, n)$ describe the structural damping of the arm.

By linearizing equation (4) around the operating point $\dot{\theta}_{0}=\ddot{\theta}_{0}=$ 0 and $\mathbf{q}_{\mathbf{0}}=\dot{\mathbf{q}}_{\mathbf{0}}=\ddot{\mathbf{q}}_{\mathbf{0}}=0$ we obtain (Geniele et al., 1995; Geniele et al., 1997):

$$
\begin{aligned}
\dot{\mathbf{v}}(t) & =\mathbf{A v}(t)+\mathbf{b} \delta \theta(t) \\
\delta y(t) & =\mathbf{c v}(t)+d \delta \theta(t)
\end{aligned}
$$

where

$$
\mathbf{v}=\left[\begin{array}{c}
\delta \mathbf{q}+\mathbf{M}^{-1} \mathbf{m} \delta \theta \\
\delta \dot{\mathbf{q}}+\mathbf{M}^{-1} \mathbf{m} \delta \dot{\theta}+\mathbf{M}^{-1} \mathbf{C} \delta \mathbf{q}
\end{array}\right]
$$

is the state vector where $\delta \mathbf{q}, \delta \dot{\mathbf{q}}, \delta \theta$ and $\delta \dot{\theta}$ denote small perturbations of $\mathbf{q}, \dot{\mathbf{q}}, \theta$ and $\dot{\theta}$ respectively around the operating point and

$$
\begin{gathered}
\mathbf{A}=\left[\begin{array}{cc}
-\mathbf{M}^{-1} \mathbf{C} & \mathbf{I}_{\mathbf{n}} \\
-\mathbf{M}^{-1} \mathbf{K}_{\mathbf{1}} & \mathbf{0}_{\mathbf{n}}
\end{array}\right] \\
\mathbf{b}=\left[\begin{array}{c}
\mathbf{M}^{-1} \mathbf{C M}^{-1} \mathbf{m} \\
\mathbf{M}^{-1} \mathbf{K}_{\mathbf{1}} \mathbf{M}^{-1} \mathbf{m}
\end{array}\right] \\
\mathbf{c}=\left[\Phi(h) \mathbf{0}_{\mathbf{1} \times \mathbf{n}}\right] \\
d=h-\Phi(h) \mathbf{M}^{-1} \mathbf{m}
\end{gathered}
$$

where

$$
\Phi(h)=\left[\phi_{1}(h) \phi_{2}(h) \cdots \phi_{n}(h)\right]
$$

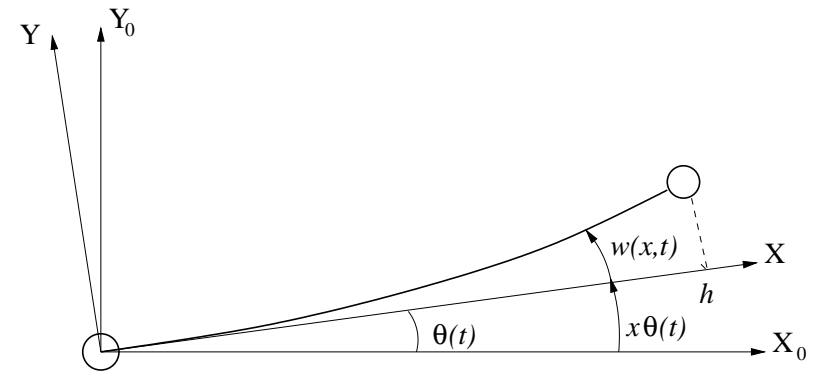

Figure 1. SKETCH OF THE FLEXIBLE LINK.

For the purpose of applying the design methodology described in the next Section it is convenient to express the system (5) in the transfer function form

$$
G(s)=\mathbf{c}(s \mathbf{I}-\mathbf{A})^{-1} \mathbf{b}+d=\frac{b(s)}{a(s)} .
$$

Note that $G(s)$ is biproper.

\section{MOTION PLANNING VIA EXACT DYNAMIC INVERSION}

The objective of the dynamic inversion based procedure is to determine a bounded input function (i.e. a motion law of the motor at the hub) which determines a desired output function (i.e. a motion law of the end-effector from a position $y_{0}$ to $y_{1}$ ). Without loss of generality, in the following we will assume $y_{0}=$ 0 . The set of all cause/effect function pairs $(\theta(\cdot), y(\cdot))$ associated to $G(s)$ be denoted by $\mathcal{B}$. The desired end-effector motion law is defined in the time interval $[0, \tau]$ by means of the "transition" polynomials introduced in (Piazzi and Visioli, 2001a):

$$
y(t ; \tau)=y_{1} \frac{(2 l+1) !}{l ! \tau^{2 l+1}} \sum_{i=0}^{l} \frac{(-1)^{l-i}}{i !(l-i) !(2 l-i+1)} \tau^{i} t^{2 l-i+1} .
$$

Outside the interval $[0, \tau], y(t ; \tau)$ is simply defined as $y(t ; \tau)=0$ if $t \leq 0$ and $y(t ; \tau)=y_{1}$ if $t \geq \tau$. In (7) the integer $l$ can be arbitrarily chosen in order to assure $y(t ; \tau) \in C^{(l)}$ over $\mathbb{R}$, i.e. $y(t ; \tau)$ has continuous derivatives up to the $h$ th order. An alternative expression of (7) is given by the integral representation (Piazzi and Visioli, 2001a)

$$
y(t ; \tau)=y_{1} \frac{(2 l+1) !}{(l !)^{2} \tau^{2 l+1}} \int_{0}^{t} v^{l}(\tau-v)^{l} d v, \quad t \in[0, \tau] .
$$

that makes clear that $y(t ; \tau)$ is monotonically increasing since $\dot{y}(t ; \tau)$ is positive over $(0, \tau)$. As a consequence, the planned motion of the end-effector is, by construction, free of oscillatory 
modes.

Note that, to select an appropriate value of $l$, the following proposition, whose proof is a straightforward consequence of the one in (Piazzi and Visioli, 1998), has to be exploited:

Proposition 1. For the system described by the transfer function (6), consider a pair $(\theta(\cdot), y(\cdot)) \in \mathcal{B}$. Then $\theta(\cdot) \in C^{(l)}$ if and only if $y(\cdot) \in C^{(l)}$ with $l$ being a non negative integer.

Hence, a suitable choice of $l$ allows to determine a continuous velocity reference function $\dot{\theta}(t)$ and in case also a continuous acceleration reference function $\ddot{\theta}(t)$.

Now, in order to perform the stable inversion, we rewrite the numerator of the transfer function (6) as follows:

$$
b(s)=b_{-}(s) b_{+}(s)
$$

where $b_{-}(s)$ and $b_{+}(s)$ denote the polynomials associated to the zeros with negative real part and positive real part respectively (appropriately, the presence of purely imaginary zeros can be excluded). Now, consider the inverse system of (6) whose transfer function can be written as:

$$
G(s)^{-1}=\gamma_{0}+H_{0}(s)
$$

where $\gamma_{0}$ is a real constant and $H_{0}(s)$, a strictly proper rational function, represents the zero dynamics. This can be uniquely decomposed according to

$$
H_{0}(s)=H_{0}^{-}(s)+H_{0}^{+}(s)=\frac{c(s)}{b_{-}(s)}+\frac{d(s)}{b_{+}(s)}
$$

where $c(s)$ and $d(s)$ are suitable polynomials. Being $\mathcal{L}$ the Laplace transform operator, define:

$$
\begin{gathered}
\eta_{0}^{-}(t):=\mathcal{L}^{-1}\left[H_{0}^{-}(s)\right] \\
\eta_{0}^{+}(t):=\mathcal{L}^{-1}\left[H_{0}^{+}(s)\right] \\
Y(s ; \tau):=\mathcal{L}[y(t ; \tau)]
\end{gathered}
$$

The unstable reference function $\theta_{u}(t ; \tau)$ that causes the desired output function $y(t ; \tau)$ can be simply determined as:

$$
\begin{aligned}
\theta_{u}(t ; \tau)= & \mathcal{L}^{-1}\left[G(s)^{-1} Y(s ; \tau)\right] \\
= & \gamma_{0} y(t ; \tau)+\int_{0}^{t} \eta_{0}^{-}(t-v) y(v ; \tau) d v+ \\
& \int_{0}^{t} \eta_{0}^{+}(t-v) y(v ; \tau) d v \\
& t \in(-\infty,+\infty) .
\end{aligned}
$$

Thus, we have that $\left(\theta_{u}(t ; \tau), y(t ; \tau)\right) \in \mathcal{B}$ and note that $\theta_{u}(t ; \tau)=0$ if $t \in(-\infty, 0)$ and $\theta_{u}(t ; \tau)$ is unbounded over $[0,+\infty)$ due to the unstable zero dynamics (associated to $H_{0}^{+}(s)$ ).

The unstable modes associated with $b_{+}(s)$ be denoted by $m_{i}(t)$, $i=1, \ldots, w$. Then, the following lemma results:

Lemma 1. There exists real constants $k_{i} \in \mathbb{R}, i=0, \ldots, w$ that depend on positive time parameter $\tau$ such that, for $t>\tau$

$$
\int_{0}^{t} \eta_{0}^{+}(t-v) y(v ; \tau) d v=k_{0}(\tau)+\sum_{i=1}^{w} k_{i}(\tau) m_{i}(t)
$$

Proof. Considering that $t>\tau$ we can rewrite the integral of the above Lemma as follows:

$$
\begin{aligned}
\int_{0}^{t} \eta_{0}^{+}(t-v) y(v ; \tau) d v= & \int_{0}^{\tau} \eta_{0}^{+}(t-v) y(v ; \tau) d v+ \\
& \int_{\tau}^{t} \eta_{0}^{+}(t-v) y_{1} d v .
\end{aligned}
$$

As it is known $\eta_{0}^{+}(t)$, the impulse response of the unstable zero dynamics can be expressed as a linear combination of the modes $m_{i}(t)$ :

$$
\eta_{0}^{+}(t)=\alpha_{1} m_{1}(t)+\cdots+\alpha_{w} m_{w}(t)
$$

where $\alpha_{i} \in \mathbb{R}, i=1, \ldots, w$ are appropriate coefficients. Taking into account the analytic expression of the transition polynomial $y(t ; \tau)$ it then follows that the integral $\int_{0}^{\tau} \eta_{0}^{+}(t-v) y(v ; \tau) d v$ is a linear combination of the modes $m_{i}(t)$ and its coefficients depend on $\tau$. On the other hand, examining the integral $\int_{\tau}^{t} \eta_{0}^{+}(t-v) y_{1} d v$ we analogously deduce that it can be expressed as a linear combination of the modes $m_{i}(t)$ plus a constant addend. Therefore, by virtue of (10) the statement of Lemma 1 follows.

At this point, taking into account Lemma 1 we can define the following function:

$$
\theta_{c}(t ; \tau):=-\sum_{i=1}^{w} k_{i}(\tau) m_{i}(t) t \in(-\infty,+\infty)
$$

The following lemma results:

Lemma 2. Being $\theta_{c}(t ; \tau)$ the function defined in (12), we have

$$
\left(\theta_{c}(t ; \tau), 0\right) \in \mathcal{B} \quad \forall \tau \in \mathbb{R}^{+}
$$

Proof. By examination of the differential equation associated to the system described by the transfer function $G(s)=$ $\left(b_{-}(s) b_{+}(s)\right) / a(s)$ it follows that the pair $\left(\theta_{c}(t ; \tau), 0\right)$ satisfies this equation over $(-\infty,+\infty)$. 
Finally, we can define the following function that performs the exact stable inversion:

$$
\theta(t ; \tau)=\theta_{u}(t ; \tau)+\theta_{c}(t ; \tau) t \in(-\infty,+\infty)
$$

The following proposition can therefore be stated.

Proposition 2. The function function $\theta(t ; \tau)$ defined in (13) is bounded over $(-\infty,+\infty)$ and $(\theta(t ; \tau), y(t ; \tau)) \in \mathcal{B}$.

Proof. Taking into account Lemma 2, evidently $(\theta(t ; \tau), y(t ; \tau)) \in$ $\mathcal{B}$ by virtue of linear superposition. On the other hand, $\theta(t ; \tau)$ is, by construction, bounded because of the exact cancellation of all the unstable modes appearing in $\theta_{u}(t ; \tau)$ (see Lemma 1 and definition (12)).

Summarizing, the determined function $\theta(t ; \tau)$ exactly solves the stable inversion problem for a family of output functions, which depend on the free transition time $\tau$. This transition time $\tau$ can be fixed by solving a simple bisection-type optimization problem whose aim is to minimize the motion time taking into account the actuators limits (Piazzi and Visioli, 2001b).

Actually, from a practical point of view, in order to use the synthesized function (13) with the chosen value $\tau^{*}$ of the transition time, it is necessary to truncate it, resulting therefore in an approximate generation of the desired output $y\left(t ; \tau^{*}\right)$. This can be done with arbitrarily precision given any couple of small parameters $\varepsilon_{0}>0$ and $\varepsilon_{1}>0$. Indeed, compute

$$
t_{0}:=\max \left\{t^{\prime} \in \mathbb{R}:\left|\theta\left(t ; \tau^{*}\right)\right| \leq \varepsilon_{0} \quad \forall t \in\left(-\infty, t^{\prime}\right]\right\}
$$

and define

$$
t_{s}:=\min \left\{0, t_{0}\right\}
$$

Similarly, compute

$$
t_{f}:=\min \left\{t^{\prime} \in \mathbb{R}:\left|\theta\left(t ; \tau^{*}\right)-\theta_{f}\right| \leq \varepsilon_{1} \quad \forall t \in\left[t^{\prime}, \infty\right)\right\}
$$

Hence, the approximate reference signal to be used is

$$
\theta_{a}\left(t ; \tau^{*}\right):= \begin{cases}0 & \text { for } t<t_{s} \\ \theta\left(t ; \tau^{*}\right) & \text { for } t_{s} \leq t \leq t_{f} \\ \theta_{f} & \text { for } t>t_{f}\end{cases}
$$

Note that $t_{s}$ depends on $\tau^{*}$ and it might occur that $t_{s}<0$, resulting in the so-called "preaction control" (Marro and Piazzi, 1996; Marro, 1996).

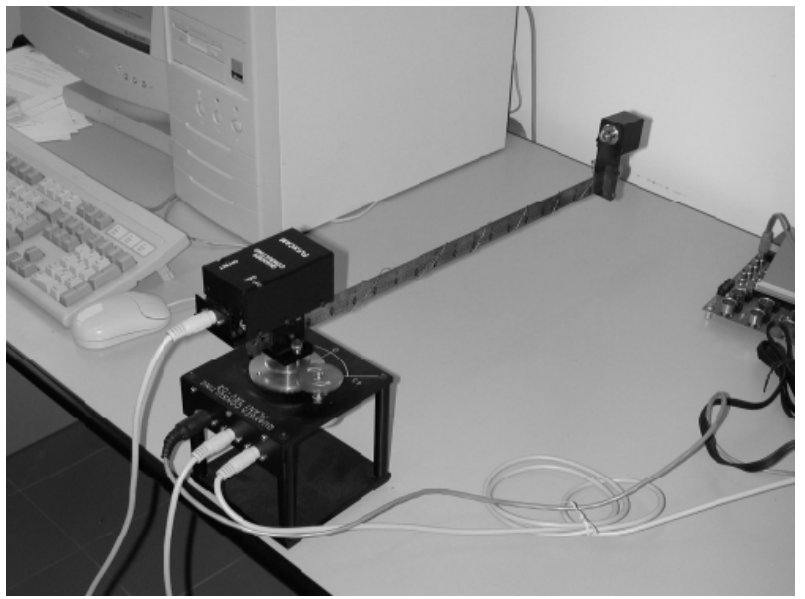

Figure 2. THE FLEXIBLE LINK ADOPTED IN THE EXPERIMENTS (AUTOMATICA LABORATORY, UNIVERSITY OF PARMA).

Table 1. VALUES OF THE PARAMETERS OF THE EXPERIMENTAL SETUP.

\begin{tabular}{ll}
\hline Parameter & Value \\
\hline Motor + Camera inertia & $0.002 \mathrm{kgm}^{2}$ \\
Link length & $0.41 \mathrm{~m}$ \\
Link height & $0.02 \mathrm{~m}$ \\
Link thickness & $0.0008 \mathrm{~m}$ \\
Link mass & $0.06 \mathrm{~kg}$ \\
Link rigid body inertia & $0.0042 \mathrm{kgm}^{2}$ \\
Payload mass & $0.05 \mathrm{~kg}$ \\
\hline
\end{tabular}

\section{EXPERIMENTAL SETUP}

The single flexible link robot adopted in the experiments is a testbed built by Quanser Consulting Inc. (see Figure 2) and available at the Automatica Laboratory of the University of Parma. The system parameters are shown in Table 1. The flexible link is actuated by means of a DC motor and a gear ratio of 1:70 is obtained by means of a pulley system. This gear ratio makes the motor somewhat, but not totally, insensitive to the arm deflections. The motor position is controlled by a PD regulator with a sampling time of $5 \mathrm{~ms}$. The tip position, which is not used in the control scheme, is measured by means of a light source, attached to the tip of the beam, which is detected by a camera mounted to the rotating base. 
Table 2. ZEROS AND POLES OF THE FLEXIBLE LINK TRANSFER FUNCTION.

\begin{tabular}{lll}
\hline Zeros & \multicolumn{1}{l}{ Poles } \\
\hline 71.34 & -50.74 & $-0.38 \pm 11.51$ \\
\hline
\end{tabular}

\section{EXPERIMENTAL RESULTS}

The methodology presented in this paper has been applied to the flexible link described in the previous section. Thus, the inversion-based technique has been adopted to determine the reference input to the motor servopositioning system. A time $\tau^{*}=0.6 \mathrm{~s}$ has been chosen to perform a motion of $0.36 \mathrm{~m}$ of the tip, that correspond to a 50 degrees motion of the hub. A fifth order transition polynomial has been selected as output function in order to ensure the continuity of the velocity and acceleration reference signal. Hence, the desired motion of the tip is:

$$
y(t)=0.36\left(\frac{6}{0.6^{5}} t^{5}-\frac{15}{0.6^{4}} t^{4}+\frac{10}{0.6^{3}} t^{3}\right) .
$$

The flexible link has been modelled as explained in the previous sections. Specifically a linear model assuming only one flexible mode has been obtained by considering the system parameters of Table 1, an estimated natural frequency of $3.9 \mathrm{~Hz}$ and a structural damping $c_{1}=0.5 \mathrm{Nm} / \mathrm{rad} \cdot \mathrm{s}^{-1}$. The poles and zeros of the resulting transfer function between the hub position and the tip position are shown in Table 2. Then, we selected $\varepsilon_{0}=\varepsilon_{1}=2 \cdot 10^{-5}$, yielding to a preaction time $t_{s}=-0.1 \mathrm{~s}$ and to $t_{f}=0.8 \mathrm{~s}$. The hub reference input $\theta(t)$ is plotted in Figure 3. The resulting tip motion is plotted in Figure 4, whilst the corresponding control signal is shown in Figure 5. For the sake of comparison, a fifth order polynomial function of $0.7 \mathrm{~s}$ has also been applied as a hub reference input (see Figure 6). The resulting motion of the endeffector is plotted in Figure 7. It is evident that the inversionbased methodology permits to greatly reducing the residual vibration effect. In particular, the maximum overshoot has been reduced from $14.4 \%$ to $1.0 \%$ and the $2 \%$ settling time has been decreased from $5.5 \mathrm{~s}$ to $0.85 \mathrm{~s}$. Furthermore, an inherent robustness to model uncertainties appears, as the system has been considered as a linear system with just one flexible mode.

\section{CONCLUSIONS}

In this paper we have presented an inversion-based methodology for the open-loop control of the end-effector of a single flexible link manipulator. Basically, it consists of determining, via an exact dynamic inversion, the motion law of the hub in order to obtain a desired non-oscillating motion of the tip. Experimental results point out the effectiveness of the methodology,

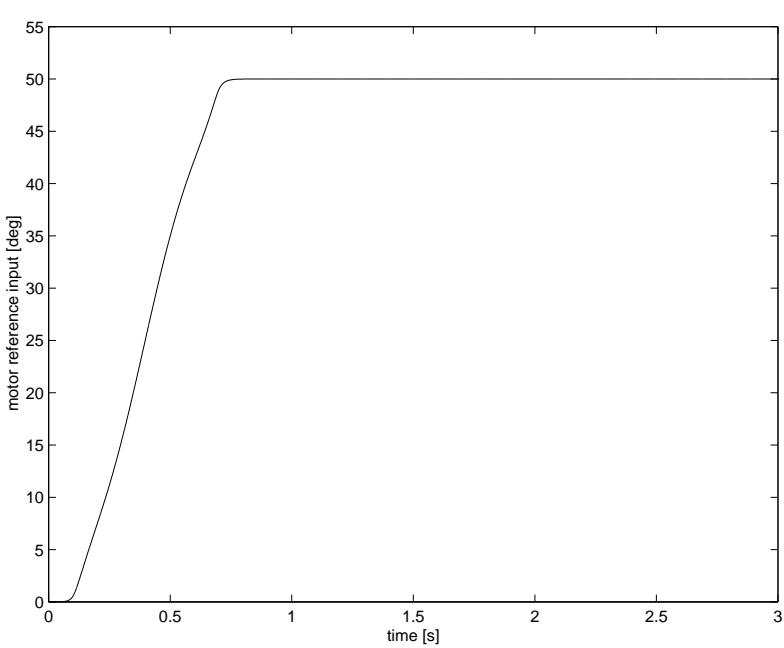

Figure 3. HUB INVERSION-BASED REFERENCE INPUT.

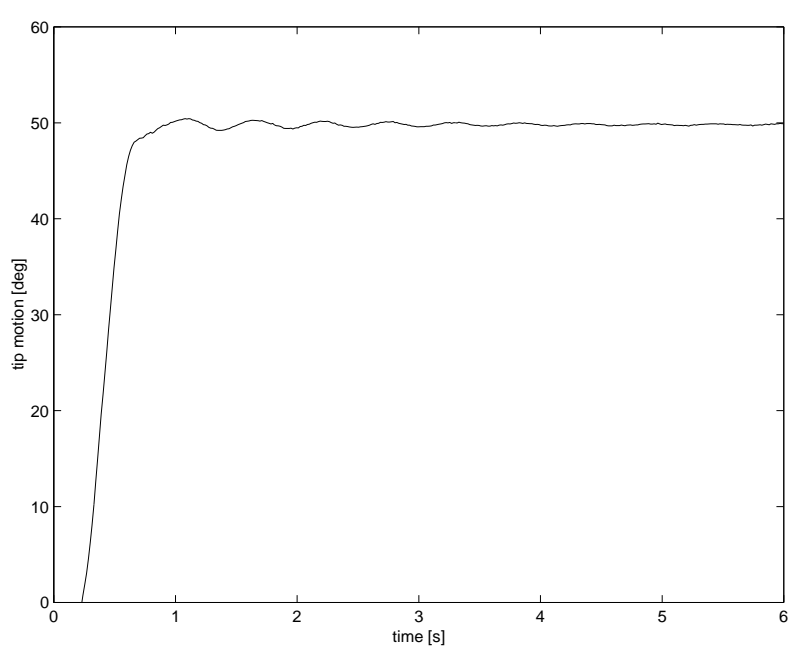

Figure 4. TIP MOTION WITH THE INVERSION-BASED TECHNIQUE.

despite it does not require a sensor to measure the tip position, and show that an inherent robustness to the model inaccuracies is achieved due to the smoothness of the adopted functions.

\section{ACKNOWLEDGMENT}

Authors wish to thank Alberto Borboni for the fruitful discussions about the modelling part and Corrado Guarino Lo Bianco, Emilio Sardini and Danilo Febbrari for their help in arranging the experimental setup.

Partial support to this research has been provided by MURST scientific research funds. 


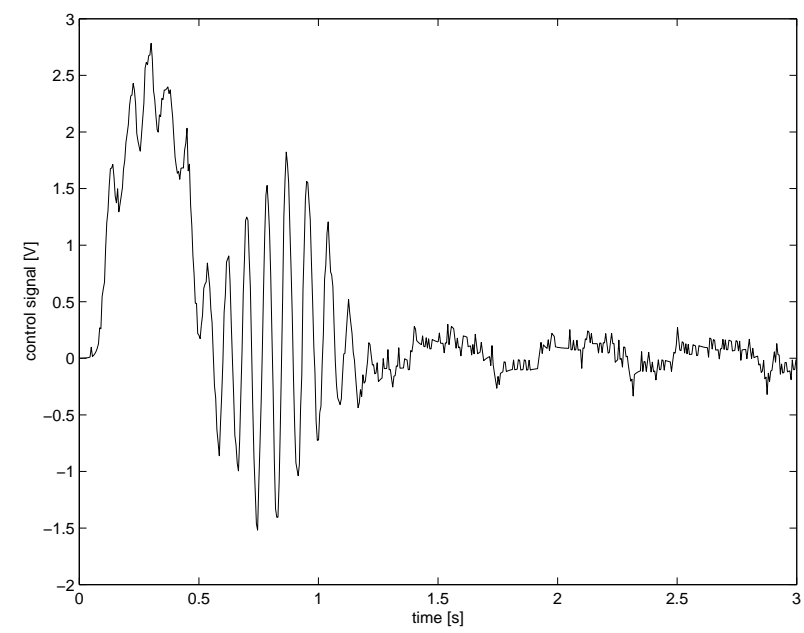

Figure 5. CONTROL SIGNAL WITH THE INVERSION-BASED TECHNIQUE.

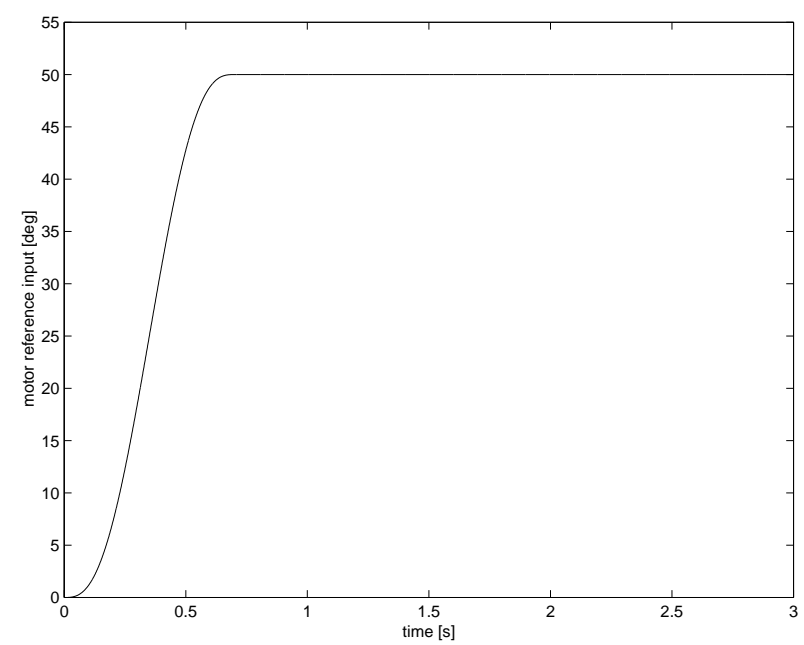

Figure 6. STANDARD POLYNOMIAL HUB REFERENCE INPUT.

\section{REFERENCES}

De Luca A., Panzieri S., Ulivi G., "Stable inversion control for flexible link manipulators", Proc. IEEE International Conference on Robotics and Automation, pp. 799-805, 1998.

Geniele H., Patel R. V., Khorasani K., "Control of a flexible link manipulator", Proc. IEEE International Conference on Robotics and Automation, pp. 1217-1222, 1995.

Geniele H., Patel R. V., Khorasani K., "End-point control of a flexible-link manipulator: theory and experiments", IEEE Transactions on Control Systems Technology, Vol. 5, No. 6, pp. 556-570, 1997.

Gross E., Tomizuka M., "Experimental flexible beam tip

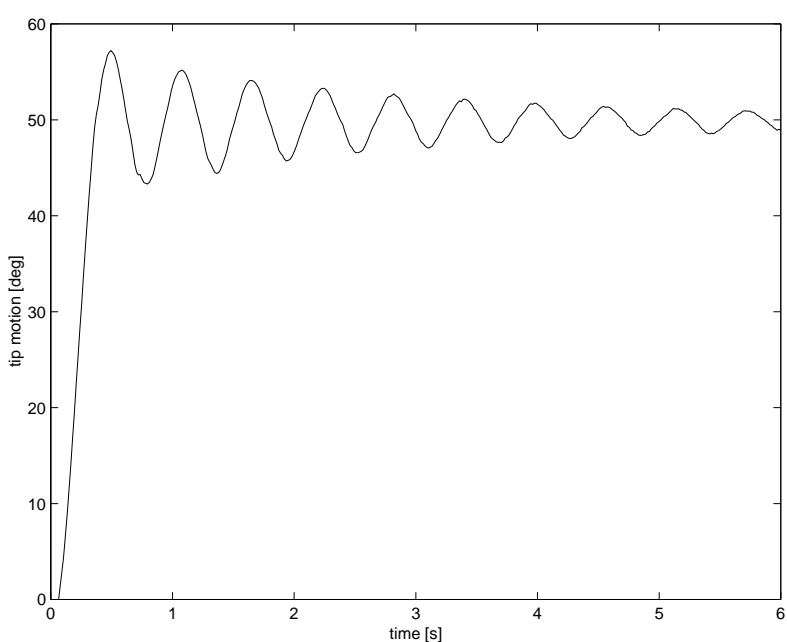

Figure 7. TIP MOTION WITH THE STANDARD TECHNIQUE.

tracking control with a truncated series approximation to uncancelable inverse dynamics", IEEE Transactions on Control Systems Technology, Vol. 2, No. 4, pp. 382-391, 1994.

Hashtrudi Zaad K., Khorasani K., "Control of non-minimum phase singularly perturbed systems with application to flexiblelink manipulators", International Journal of Control, Vol. 63, pp. 679-701, 1996.

Meirovitch L., Analytical methods in vibrations, Macmillan, New York, 1967.

G. Marro, A. Piazzi, "A geometric approach to multivariable perfect tracking", Proc. of the 13th IFAC World Congress, pp.241-246, 1996.

G. Marro, "Multivariable regulation in geometric terms: old and new results", in Colloquium on Automatic Control, C. Bonivento, G. Marro and R. Zanasi eds, Springer-Verlag, pp. 77138, 1996.

Menini L., Tornambe A., Zaccarian L., "Flexible robots", in Modelling and control of mechanisms and robots, C. Melchiorri and A. Tornambe eds, World Scientific, Singapore, pp. 115-148, 1996.

Moallem M., Patel R. V., Khorasani K., "An inverse dynamics control strategy for tip position tracking of flexible multi-link manipulators", Journal of Robotic Systems, Vol. 14, No. 9, pp. 649-658, 1997.

Panzieri S., Ulivi G., "Disturbance rejection of iterative learning control applied to trajectory tracking for a flexible link manipulator", Proc. European Control Conference, pp. 23742379, 1995.

Piazzi A., Visioli A., "Minimum-time open-loop smooth control for point-to-point motion in vibratory systems", Proc. IEEE International Conference on Robotics and Automation, pp. 946-951, 1998. 
Piazzi A., Visioli A., "Minimum-time system-inversionbased motion planning for residual vibration reduction", IEEE/ASME Transactions on Mechatronics, Vol. 5, No. 1, pp. 12-22, 2000.

Piazzi A., Visioli A., "Optimal noncausal set-point regulation of scalar systems", Automatica, Vol. 37, No. 1, pp. 121-127, 2001.

Piazzi A., Visioli A., "Robust set-point constrained regulation via dynamic inversion", International Journal of Robust and Nonlinear Control, Vol. 11, pp. 1-22, 2001.

TalebiH. A., Patel R. V., Khorasani K., "Inverse dynamics control of flexible-link manipulators using neural networks", Proc. IEEE International Conference on Robotics and Automation, pp. 806-811, 1998.

Talebi H. A., Khorasani K., Patel R. V., "Experimental results on tracking control of a flexible-link manipulator: a new output redefinition approach", Proc. IEEE International Conference on Robotics and Automation, pp. 1090-1095, 1999.

Wang D., Vidyasagar M., "Transfer functions for a single flexible link", Proc. IEEE International Conference on Robotics and Automation, pp. 1042-1047, 1989.

Wang D., Vidyasagar M., "Transfer functions for a single flexible link", The International Journal of Robotic Research, Vol. 10, No. 5, pp. 540-549, 1991.

Yang H., Krishnan H., Ang M. H., "Tip-trajectory tracking control of single-link flexible robots via output redefinition", Proc. IEEE International Conference on Robotics and Automation, pp. 1102-1107, 1999. 